\title{
Cell-specific epigenetic modulation
}

The targeted delivery of a DNA methyltransferase inhibitor to either $\mathrm{CD}^{+}$or $\mathrm{CD}^{+} \mathrm{T}$ cells can ameliorate lupus-like disease in mice, according to new findings published in JCI Insight. These findings highlight the importance of DNA hypermethylation in systemic lupus erythematosus (SLE) and the therapeutic potential of cell-specific inhibition of DNA methylation.

$\mathrm{T}$ cells are major contributors to SLE pathogenesis, and T cell abnormalties (such as a defective regulatory $\mathrm{T}$ cell and/or expanded double-negative $\mathrm{T}$ cell compartment) are common in patients.

"Previous work suggests that global demethylation defects in patients with SLE contribute to the development of autoimmunity and pathology", explains George Tsokos, corresponding author of the study. "We hypothesized that demethylation affects the function of various immune cell subsets differently."
The researchers delivered a demethylating agent (5-azacytidine; 5-Aza) to specific T cell subsets using nanolipogels coated with either anti-CD4 or anti-CD8 antibodies. CD4-specific DNA demethylation resulted in the expansion of regulatory $\mathrm{T}$ cells, whereas CD8-specific DNA demethylation resulted in a decrease in the generation of double-negative $\mathrm{T}$ cells in mice. Both approaches ameliorated established disease in lupus-prone mice, unlike treatment with unloaded nanolipogels or with free 5-Aza.

"Previous data from our group suggest that $\mathrm{CD} 8^{+} \mathrm{T}$ cells convert to double-negative $\mathrm{T}$ cells through transcriptional silencing of CD $8 \alpha$ and CD $8 \beta$," says Hao Li, first author on the study. "Loss of CD8 expression on $\mathrm{CD}^{+} \mathrm{T}$ cells could lead to a reduction in cytotoxic capacity, which is important for controlling the development of autoimmunity and lupus-like disease."

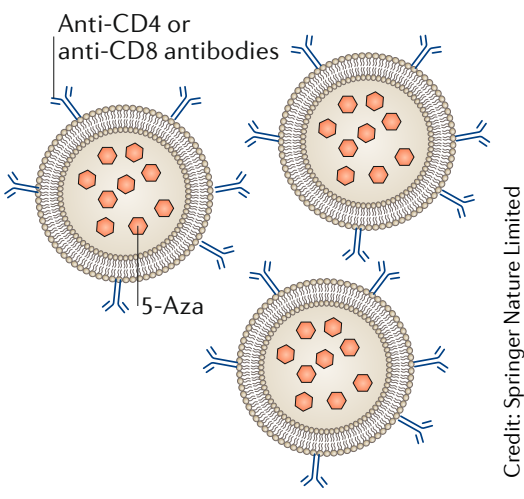

In a mouse model of T cell-mediated autoimmunity using adoptively transferred T cells, they detected a simultaneous loss of both CD8 and perforin expression in the $\mathrm{CD} 8^{+}$ T cells, which was inhibited with 5-Aza (targeted to the transferred T cells).

"Our findings suggest that the role of DNA methylation in SLE development is rather complex," remarks Li. "We would like to apply this approach to other immune cell subsets known to contribute to SLE pathogenesis including macrophages, B cells, and tissue resident cells," concludes Tsokos.

Jessica McHugh of doublenegative $\mathrm{T}$ cells

ORIGINAL ARTICLE Li, H. et al. Precision DNA demethylation ameliorates disease in lupus-prone mice. JCl Insight https://doi.org/10.1172/ jci.insight.120880 (2018)

\section{CSF1 drives pain \\ in arthritis}

Colony-stimulating factor 1 (CSF1; also known as M-CSF) is involved in the development of cells of the monocyte-macrophage lineage, which have an important function in the pathogenesis of rheumatoid arthritis (RA). The results of a new study suggest that CSF1 also has a role in the development of pain in models of inflammatory arthritis.

"We and others have shown previously that CSF1 can be a proinflammatory cytokine, and there have been clinical trials in RA targeting CSF1 or its receptor," explains corresponding author John Hamilton. "Given this data and the observation that central targeting of CSF1 was effective in treating neuropathic pain, we decided to ascertain whether peripheral CSF1 blockade with a monoclonal antibody would be effective in reducing inflammatory and arthritic pain."

To do so, Hamilton and colleagues used both lymphocyte-independent (induced by zymosan, granulocyte-macrophage colony-stimulating factor (GM-CSF) or TNF) and lymphocyte-dependent (induced by antigen or IL-1 $\beta$ ) mouse models of inflammatory arthritis. Mice were treated with antibodies against CSF1 and/or its receptor either before or after induction of disease, and pain was assessed using an incapacitance meter. "This method is particularly relevant for the measurement of knee pain, since less weight is placed on an arthritic limb," says Hamilton.

Blockade of CSF1 prevented the development of pain and reduced disease severity in lymphocyte-independent models of arthritis compared with control mice, but did not ameliorate established disease or pain in these models.

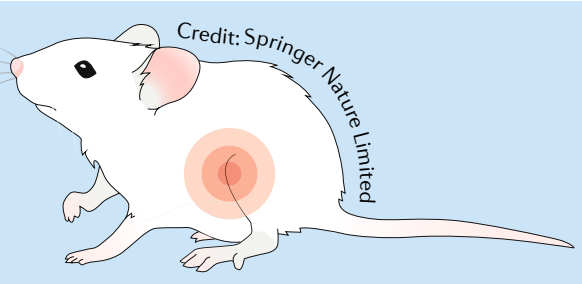

Conversely, blockade of CSF1 had no effect on pain or disease in lymphocytedependent models of arthritis.

Interestingly, systemic administration of CSF1 induced pain and disease in an already inflamed joint. However, the mechanism by which CSF1 functioned differed from that of GM-CSF and TNF.

"These results suggest that CSF1 might only be relevant when the driving inflammatory insults in tissues are acute and/or periodic," states Hamilton. "In future studies, we plan to assess the generality of our findings for the role of

systemic administration of CSF 1 induced pain and disease
Joanna Collison

ORIGINAL ARTICLE Saleh, R. et al. CSF-1 in inflammatory and arthritic pain development. J. Immunol. https://doi.org/10.4049/jimmunol. 1800665 (2018) 